\title{
Implementation of Predictive Data Mining Techniques for Identifying Risk Factors of Early AVF Failure in Hemodialysis Patients
}

\author{
Mohammad Rezapour, ${ }^{1}$ Morteza Khavanin Zadeh, ${ }^{2}$ and Mohammad Mehdi Sepehri ${ }^{1,3}$ \\ ${ }^{1}$ Department of Industrial Engineering, Tarbiat Modares University, Tehran 14117-13114, Iran \\ ${ }^{2}$ Hasheminejad Clinical Research Development Center (HCRDC) and Rasoul Akram Hospital, \\ Tehran University of Medical Sciences (TUMS), Iran \\ ${ }^{3}$ Hospital Management Research Center, Iran University of Medical Sciences, Tehran 19697-14713, Iran
}

Correspondence should be addressed to Morteza Khavanin Zadeh; m-khavanin@tums.ac.ir

Received 11 January 2013; Revised 2 March 2013; Accepted 14 March 2013

Academic Editor: Enzo Grossi

Copyright ( 2013 Mohammad Rezapour et al. This is an open access article distributed under the Creative Commons Attribution License, which permits unrestricted use, distribution, and reproduction in any medium, provided the original work is properly cited.

\begin{abstract}
Arteriovenous fistula (AVF) is an important vascular access for hemodialysis (HD) treatment but has 20-60\% rate of early failure. Detecting association between patient's parameters and early AVF failure is important for reducing its prevalence and relevant costs. Also predicting incidence of this complication in new patients is a beneficial controlling procedure. Patient safety and preservation of early AVF failure is the ultimate goal. Our research society is Hasheminejad Kidney Center (HKC) of Tehran, which is one of Iran's largest renal hospitals. We analyzed data of $193 \mathrm{HD}$ patients using supervised techniques of data mining approach. There were 137 male (70.98\%) and 56 female (29.02\%) patients introduced into this study. The average of age for all the patients was $53.87 \pm$ 17.47 years. Twenty eight patients had smoked and the number of diabetic patients and nondiabetics was 87 and 106, respectively. A significant relationship was found between "diabetes mellitus," "smoking," and "hypertension" with early AVF failure in this study. We have found that these mentioned risk factors have important roles in outcome of vascular surgery, versus other parameters such as "age." Then we predicted this complication in future AVF surgeries and evaluated our designed prediction methods with accuracy rates of $61.66 \%-75.13 \%$.
\end{abstract}

\section{Introduction}

Chronic kidney disease $(\mathrm{CKD})$ is a condition in which the kidneys are damaged and cannot filter blood as well as possible. In advanced stage of CKD known as end-stage renal disease (ESRD) kidney functions are reduced very severely. Hemodialysis (HD) treatment is the most common procedure which is performed for ESRD patients and $\mathrm{HD}$ requires permanent vascular access (VA) as an important aspect [1]. Furthermore, there are three main types of VA used in HD treatment: arteriovenous fistula (AVF), synthetic arteriovenous graft (AVG), and central venous catheter (CVC) [2]. Types of VA have their risks and can be expensive. According to recommendation of clinical practice guidelines, AVF is the access of first choice based on the reduced associated complications, morbidity, and mortality compared with AVG and CVC; also AVF has superior survival rate (estimated at $90 \%$ after one year) than other VA types (such as $60 \%$ of AVG) [3]. AVF is less expensive and remains the gold standard access to HD.

Since incidence of early AVF failure is reported as $20-60 \%$ [4], therefore detection of risk factors in early AVF failure is essential in caring for these HD patients in terms of medical, economic, and psychological impact. An AVF that is never usable for dialysis or that fails within three months of usage, should be classified as an early failure [5]. The goals of our study are to extract pattern of early AVF failure, predict this issue, and determine the high-risk factors on it, using data mining approaches.

"Data mining" is defined as a step in the knowledge discovery in databases (KDD) process that consists of applying data analysis and discovery algorithms that, under acceptable 
computational efficiency limitations, produce a particular enumeration of patterns (or models) over the data [6]; also KDD is defined as the nontrivial process of identifying valid, novel, potentially useful, and ultimately understandable patterns in data. Data mining's approaches are boosting and their applications have become increasingly essential for healthcare organizations to make decisions based on the analysis of the huge amounts of clinical data generated by healthcare transactions. Data mining is becoming increasingly popular in healthcare, if not increasingly essential, and several factors motivated the use of data mining applications in healthcare, such as fraud and abuse detection, ability of transforming data, and benefit of healthcare providers [7]. Another factor is that data mining can improve decision making by discovering patterns and trends in large amounts of complex data [8]. Several studies employed data mining approaches to discover the knowledge of relation between the measured parameters and prevention of AVF failure. Temporal data mining techniques are studied [9] for dialysis failure prediction and analyzing the data of dialysis sessions coming from 43 different patients. $K$-means and expectation maximization algorithm are implemented [10] to cluster some attributes of HD patients. Data mining is considered in the medical settings of HD treatment and provided a brief review of state-of-the-art methods for predicting patient risk and survival of dialysis patients [11]. WJ.48 tree algorithm is used for analyzing data of 170 patients on dialysis for 12 or more months and interpreted patterns of high-risk groups in patients by extracting decision rules [12].

1.1. World Statistics. The prevalence of CKD is increasing around the world. In 2010, more than $10 \%$ of people or more than 20 million patients in the USA have CKD [13]. According to recent statistics of National Kidney Foundation (NKF), there are 26 million CKD patients in USA in 2012 [14]. Also the rate of ESRD as a chronic illness has grown rapidly in recent years [15]. At the end of 2004, ESRD is reported with a prevalent world population of 400,000, including over 300,000 hemodialysis (HD) patients [16]. At the end of 2008, 547,982 USA residents were under treatment of ESRD and 382,343 of them received dialysis, of which 354,443 were under hemodialysis [17].

In Iran more than 14,000 patients are treated with chronic HD therapy for ESRD [18]. AVF is used by $93.4 \%$ of Iranian HD patients [1]. This finding exceeds the recommendations by guidelines and the percentage of $67-91 \%$ reported by AVF usage in many Western countries in recent years [19].

\section{Materials and Methods}

2.1. Patient Population. This study comprises AVF data of 193 patients who were under hemodialysis (HD) in Hasheminejad Kidney Center (HKC) of Tehran, which is one of Iran's largest renal hospitals. There were 137 male (70.98\%) and 56 female $(29.02 \%)$ patients introduced into this study. The average of age for all the patients was $53.87 \pm 17.47$ years. Twenty eight patients had smoked and the number of diabetic patients and nondiabetics was 87 and 106, respectively. In previous study, we clustered the attributes of early AVF failure, using descriptive approaches with $n=99$ patients [20].

In present study, we have two datasets of this vascular surgery: one of datasets was collected during period from year 2005 to 2006 and contains 36 parameters of 99 patients who all had early AVF failure; second dataset was collected from December to November 2010 and contains 25 parameters of 94 patients of whom 87 patients had survival surgery and the remaining (7 patients) had early AVF failure. So, by merging these two datasets we have 106 patients with early AVF failures and 87 patients without AVF failure.

2.2. Data Parameters. We merged two datasets and found eight similar parameters of them, where each patient is characterized by seven attributes: age (age of patients), sex (male or female), htn (hypertension), DiabetesM (diabetes mellitus), Hgb (hemoglobin), smoking (yes or no), and location (site of AVF with brachial or radial). The last column thrombosis (yes: failure or no: survival) is the designated class attribute. So we obtained a final dataset with 8 parameters of 193 records (patients), which contain 106 cases of failures and 87 cases of survivals.

2.3. Methodologies. In present study, we use supervised techniques of data mining to predict early AVF failure and determine the risk factors that have important roles on it. Many numbers of possible choices in Weka operators are available; we performed many types of them and consulted the surgeon about obtained decision trees (DT). Our choosing of the operator was on the bases that its extracted DT contains new knowledge for help in next vascular surgeries and finally due to the best accuracy rate between prediction methods (as we will see in part of "validation of analyses"). Sampling data for predictions (and number of training patients) are selected randomly. We present a brief description of the learner algorithms used in this work: JRIP and J48.

JRIP rule learner was proposed by Cohen as an optimized version of learning algorithm IREP (incremental reduced error pruning); JRIP implements repeated incremental pruning to produce error reduction (RIPPER) in Java, a prepositional rule learner [21]. Rules are created for every class in the training set and are then pruned. In this algorithm, the discovered knowledge is represented in the form of IF-THEN prediction rules, which have the advantage of being a highlevel and symbolic knowledge representation contributing towards the comprehensibility of the discovered knowledge [22]. The method is based on the construction of a rule set in which all positive examples are covered. Initially, the current set of training examples are partitioned into two subsets, a growing set and a pruning set. The rule is constructed from examples in the growing set. The rule set initiates with an empty rule set and rules are added incrementally to the rule set until no negative examples are covered. After that, JRIP replaces or revises individual rules by using reduced error pruning in order to increase the accuracy of rules. It replaces or revises individual rules by using reduced error pruning. To prune a rule the algorithm takes in account only a final 
TABLE 1: The extracted rules with JRIP learner ("yes" means early AVF failure).

\begin{tabular}{ll}
\hline JRIP rules & \\
$\begin{aligned}(\text { DiabetesM }=\text { yes }) & \rightarrow \text { Failure }=\text { no }(87.0 / 37.0) \\
& \rightarrow \text { Failure }=\text { yes }(106.0 / 37.0)\end{aligned}$ \\
Number of rules: 2
\end{tabular}

sequence of conditions from the rule and sorts the deletion that maximizes the function [23].

J48 decision tree is an implementation of the well-known Quinlan algorithm (C4.5) [24], which is an improvement algorithm, derived from basic ID3 induction system using the standard TDIDT (top-down induction of decision trees) approach, recursively partitioning the data into smaller subsets based on the value of an attribute [24, 25]. The "pruned" version of J48 reduces the chances of overfitting the data [26]. This classifier builds a decision tree for the given dataset, whose nodes represent discrimination rules acting on selective features by recursive partitioning of data, using depth-first strategy. The algorithm uses the fact that each attribute of the data can be used to make a decision by splitting the data into smaller subsets. To make the decision, the algorithm considers all the possible tests that can split the dataset and culls a test that gives the highest information gain. For each discrete attribute, one test with outcomes for each distinct value of the attribute is considered. For each continuous attribute, binary tests involving every distinct value of the attribute are considered. In order to gather the entropy gain of all these binary tests efficiently, the training data set belonging to the node in consideration is sorted for the values of the continuous attribute and the entropy gains of the binary cut based on each distinct values are calculated in one scan of the sorted data. Then a new feature is chosen and the splitting process is repeated for each attribute in a recursive manner until further splitting is not gainful. In the resulting tree structure; each inner node in the tree corresponds to an attribute, each branch represents a possible value or range of values of that attribute and each leaf represents the predicted value of target attribute [23].

The numbers in (parentheses) at the end of each leaf tell us the number of examples in this leaf. If one or more leaves were not pure (= all of the same class), the number of misclassified examples would also be given, after a slash (“"”) [27].

2.3.1. Rule Mining. At first, we analyze on seven parameters, without considering side of AVF in data (location attribute) in this stage. We extract the rules embedded in data, using "JRIP" operator. This classifier implements a propositional rule learner from data. The obtained rules in Table 1 show a determining role of diabetes mellitus (DM) in AVF failure.

The extracted tree after running J48 over the data shows smoking and DM as risk factors (Figure 1).

The second operator is "rule learner" and its results are shown in Table 2.

If we consider the parameter "location" of AVF in our analysis, the extracted rules are shown in Table 3. Also from
TABLE 2: The extracted rules by running "rule learner."

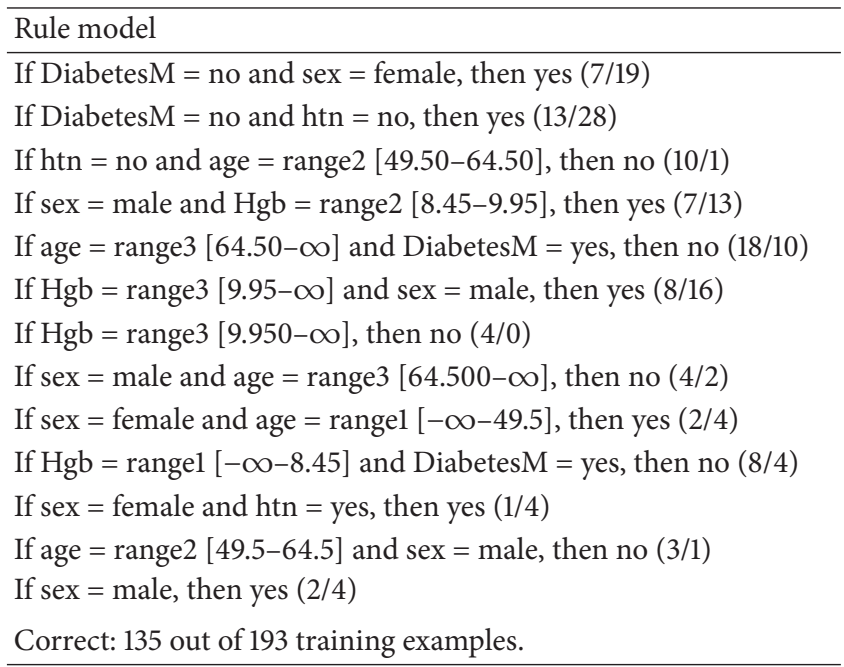

TABLE 3: Extracted rules, analysis with side of AVF.

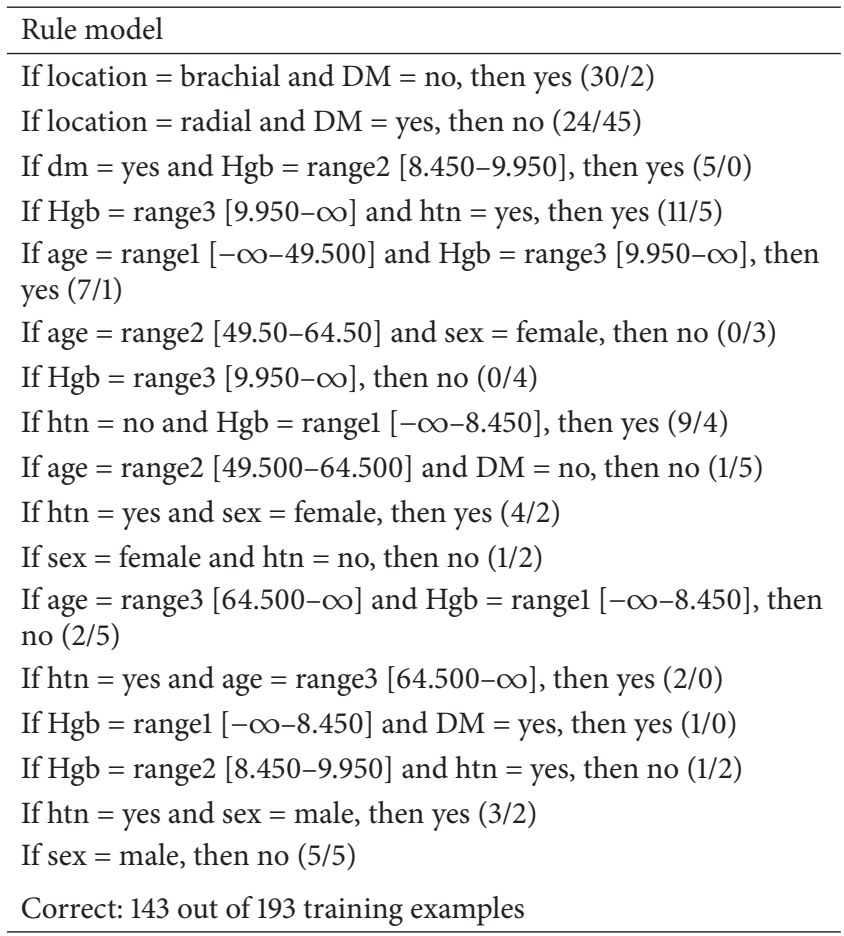

Tables 2 and 3 it seems that "htn" (hypertension) is a very important parameter.

Prediction I. Now we select training data, from original data by "stratified sampling" method. For this purpose, we choose sample_ratio $=0.1$; then 20 samples are selected, where 11 numbers of them were in class "yes" and 9 numbers in class "no." The rules that are hidden in this part of data will be detected after system training using decision tree, as shown in Figure 2.

Therefore in above selected data, three parameters have important roles: age, smoking, and diabetes. Now we predict 


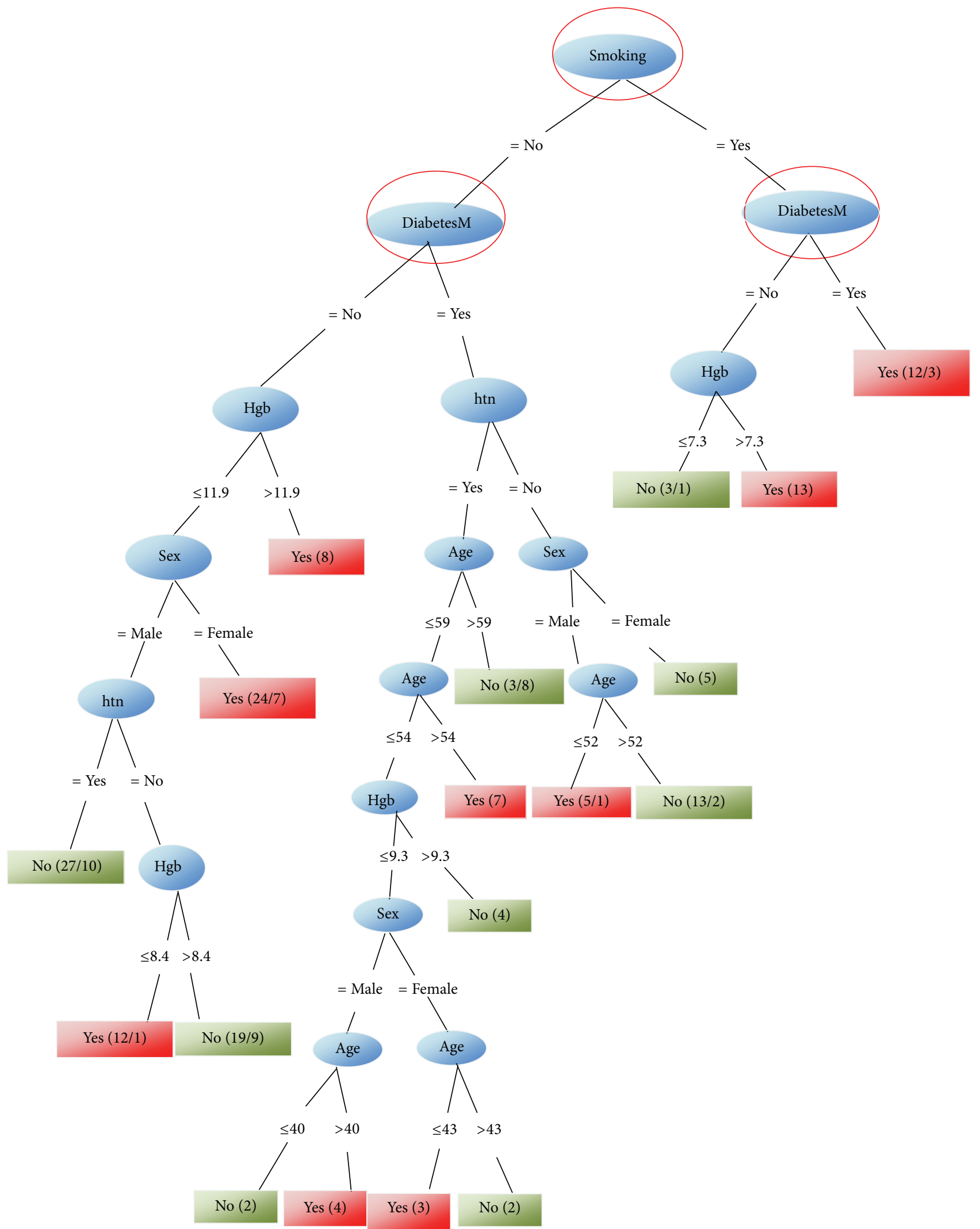

FIGURE 1: Effects of diabetes and smoking are determined from interpreted rules of this tree. 


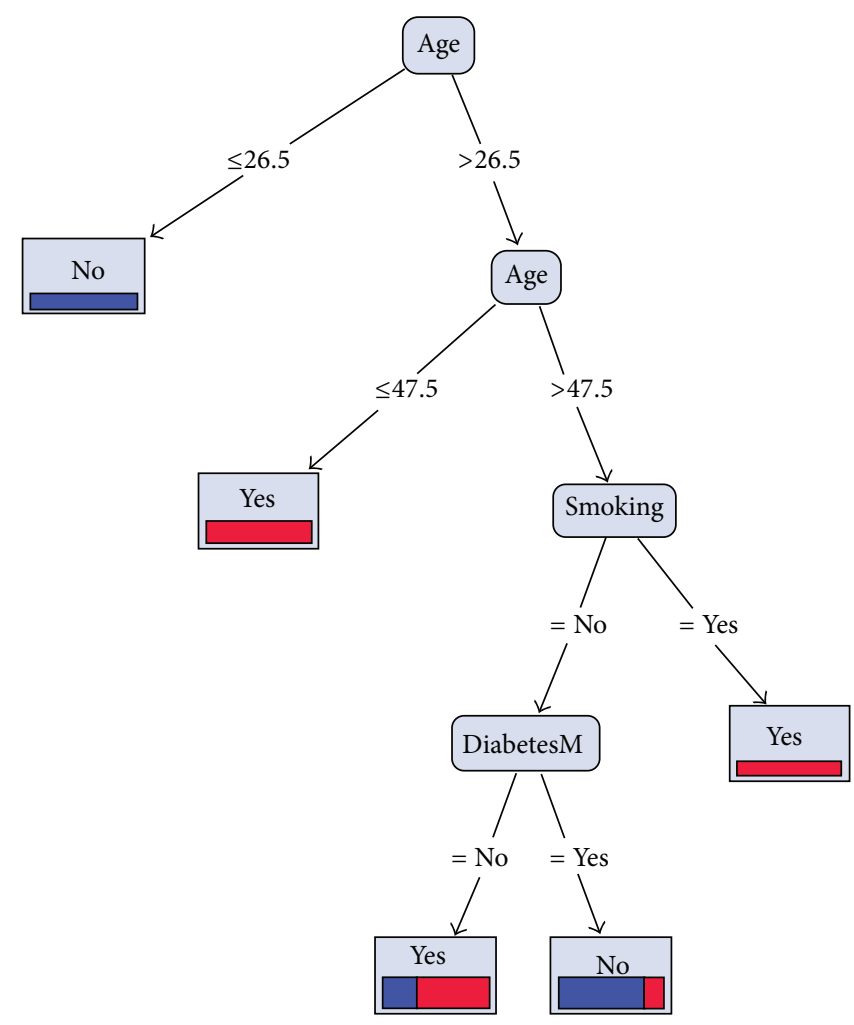

FIgURE 2: Decision tree of system training, after stratified sampling.

the AVF failure here as the first method, as shown in Table 4. So, the new field "prediction (failure)" will be created.

Prediction II. In the second method by "absolute stratified sampling" and system training with J48 tree, we finalize the tree of Figure 3.

Thus the AVF failure prediction is due to Table 5.

Comparing the predicted results in Tables 4 and 5 shows that the results of method II are nearer to the real events. We will see this fact in assessment section exactly; also here we denote that in corresponding figures with recent tables, Figure 3 is more compatible with medical experiences of the surgeon. Hence we prefer Figure 3 over Figure 2 and follow that role of "smoking" and "diabetes" (in Figure 3) is more effective than that of "age" (in Figure 2) as high-risk factors in early AVF failure.

\section{Results}

Existence of either diabetes mellitus or smoking in HD patients increases early AVF failure in their surgery. We designed two applied methods and at first predicted risk factors of this complication with accuracy rates of $61.66 \%-$ $74.61 \%$. Then we added data of side of AVF (location in hand) to the data and predicted this complication with accuracy rates between $67.91 \%$ and $75.13 \%$. Results support the impressive roles of risk factors in AVF failures. We found that "diabetes mellitus," "smoking," and "hypertension" have important roles in early AVF failure, which are more effective roles than other factors such as "age."

3.1. Approaches Assessment. In our study, as described previously, the separate effects of diabetes, smoking, and hypertension, respectively, are determined in accordance with the extracted rules (in Tables 1-3). Also the role of them together can be found in Figures 1-3. As we described earlier, we designed two methods of sampling and system training and analyzed them. Furthermore we measured the accuracy rates of these methods and saw their rates between $61.66 \%$ and 74.61\% (Tables 6 and 7).

In the first method ("stratified sampling" and system training with decision tree), we have the accuracy rate equal to $61.66 \%$ (Table 6).

Also the accuracy rate of method 2 will measure equal to 74.61\% (Table 7).

Therefore due to accuracy rates, the predicted results in the 2 nd method are better approximated than those of the 1st method. Therefore we denote that Diabetes and then smoking are more effective than age in AVF failure (i.e., higher factor in tree of method 1).

Finally, we added the surgery side parameter (location) and also we surveyed two other methods using "neural network" and "naïve Bayesian." Then we predicted early AVF failure with better accuracy rates of $67.91 \%-75.13 \%$ which says an important role of the "location." So we obtained 


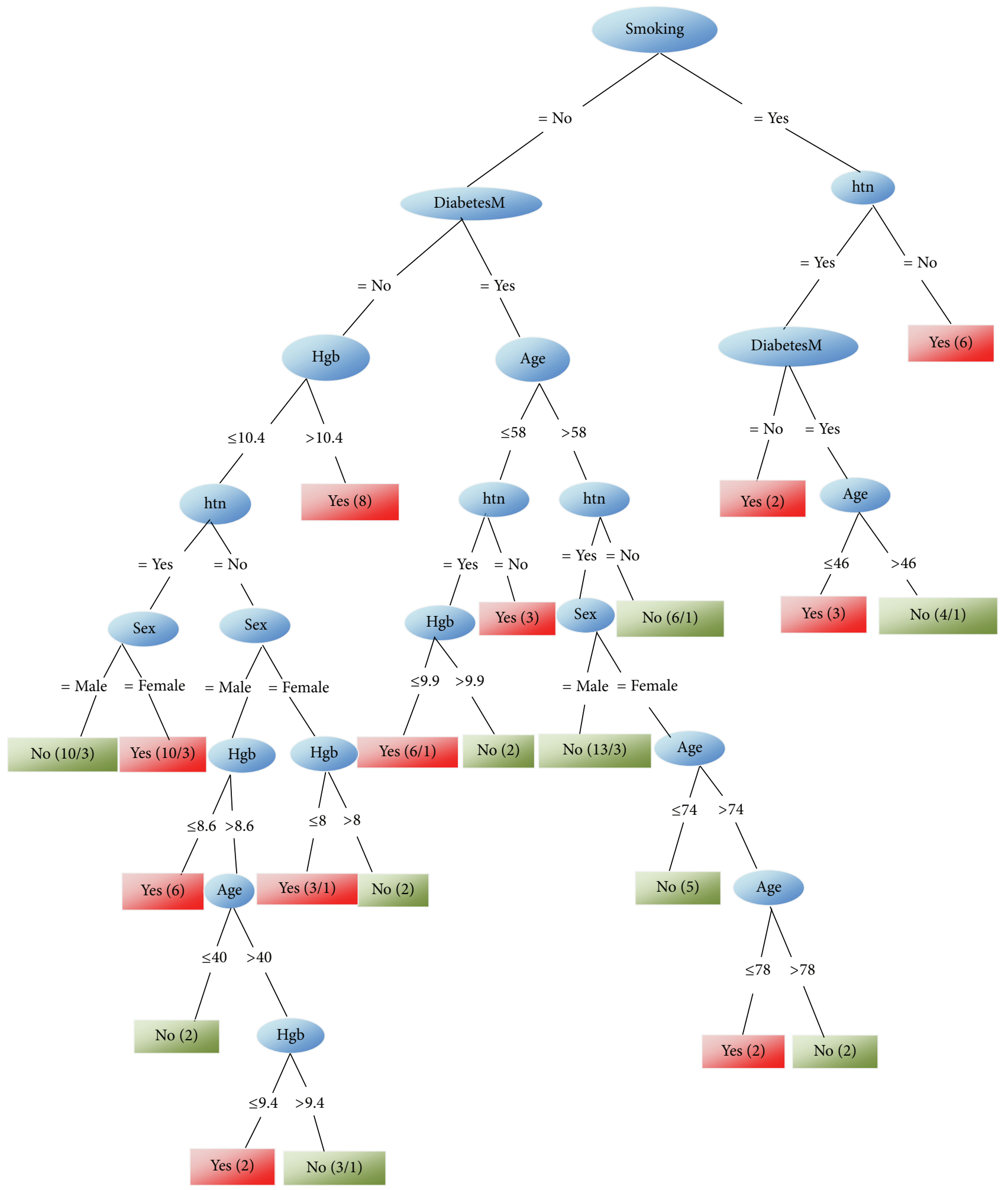

FIGURE 3: System training by J48 tree, after absolute stratified sampling. 
TABLE 4: The prediction results by method one.

\begin{tabular}{lccc}
\hline Id & Integer & Avg = 97 \pm 55.714 & {$[1.0 ; 193.0]$} \\
\hline Failure & Nominal & Mode = yes (106), least = no (87) & No (87), yes (106) \\
Prediction (failure) & Nominal & Mode = yes (112), least = no (81) & No (81), yes (112) \\
Confidence (no) & Real & Avg $=0.457 \pm 0.380$ & {$[0.0 ; 1.0]$} \\
Confidence (yes) & Real & Avg $=0.543 \pm 0.380$ & {$[0.0 ; 1.0]$} \\
\hline
\end{tabular}

TABLE 5: The prediction results by the 2 nd method.

\begin{tabular}{lccc}
\hline Id & Integer & Avg = 97 \pm 55.714 & {$[1.0 ; 193.0]$} \\
\hline Failure & Nominal & Mode = yes (106), least = no (87) & No (87), yes (106) \\
Prediction (failure) & Nominal & Mode = yes (105), least = no (88) & No (88), yes (105) \\
Confidence (no) & Real & Avg $=0.408 \pm 0.371$ & {$[0.0 ; 1.0]$} \\
Confidence (yes) & Real & Avg $=0.592 \pm 0.371$ & {$[0.0 ; 1.0]$} \\
\hline
\end{tabular}

TABLE 6: The accuracy rate in method one.

\begin{tabular}{lccc}
\hline & \multicolumn{3}{c}{ Accuracy: 61.66\% } \\
& True no & True yes & Class precision \\
\hline Pred. no & 47 & 34 & $58.02 \%$ \\
Pred. yes & 40 & 72 & $64.29 \%$ \\
Class recall & $54.02 \%$ & $67.92 \%$ & \\
\hline
\end{tabular}

TABLE 7: The accuracy rate in method two.

\begin{tabular}{lccc}
\hline & \multicolumn{3}{c}{ Accuracy: $74.61 \%$} \\
& True no & True yes & Class precision \\
\hline Pred. no & 63 & 25 & $71.59 \%$ \\
Pred. yes & 24 & 81 & $77.14 \%$ \\
Class recall & $72.41 \%$ & $76.42 \%$ & \\
\hline
\end{tabular}

the numbers of "predicted patients with/without early AVF failure" and rate of any recent methods in Table 8 .

To compare above results with real end points, remember that we had 193 patients with 106 cases of failure and 87 cases of survival of AVF.

\section{Discussion and Conclusions}

Extracting potential knowledge from vascular surgery information is showed using data mining techniques. In the proposed analysis of early AVF fistula phenomenon we can find vulnerable patients and determine decision about using AVF or replace it by other methods as AVG. Ultimate benefits are diagnosed and control risk factors of patients or making recommendations for them.

In present study we detected the high-risk factors and predicted the risk of AVF early failure in patients, using supervised techniques. These approaches give a better functionality to expert system of hospitals. Scientific prediction and control of the AVF failure can help achieving the target of percent AVF use in the prevalent HD population. Additionally, the prediction of AVF failure has a major role in the planning in these cases: if the percentage of failure was high, they may use other surgery methods such as the AVG; if the risk of
TABLE 8: Final predicted results in analysis with location.

\begin{tabular}{llcc}
\hline $\begin{array}{l}\text { Sampling } \\
\text { method }\end{array}$ & Training algorithm & \multicolumn{2}{c}{ Prediction results } \\
\hline Stratified & Neural network & Accuracy: & $69.97 \%( \pm 5.17 \%)$ \\
& & Yes/no: & $74 / 119$ \\
\multirow{2}{*}{ Stratified } & Decision tree & Accuracy: & $67.91 \%$ \\
& & Yes/no: & $94 / 99$ \\
\multirow{2}{*}{ Stratified } & \multirow{2}{*}{ Naïve Bayesian } & Accuracy: & $69.97 \%( \pm 8.61 \%)$ \\
& & Yes/no: & $100 / 93$ \\
Absolute & \multirow{2}{*}{ WJ-48 } & Accuracy: & $75.13 \%$ \\
\hline
\end{tabular}

fistula failure was low, they may use the same method (AVF) but make recommendations to the patient and his/her family, such as at what level they should keep the blood pressure of the patient after surgery.

We examined the risk factors of early AVF failure in vascular access surgery. The research was studied in a real society of hemodialysis (HD) patients and we concluded that diabetes, smoking, and hypertension then are risk factors of early AVF failure. We used data mining techniques and designed prediction approaches to predict probability of this complication in new HD patients whom have been referred by nephrologists to AVF surgery.

In conclusion, these outcomes enable early detection of those patients that have a higher risk and can guide the surgeon to select the surgical procedure. Moreover, our additional clinical sequent is that proposes the decision about selecting more useful vascular access method to guide the surgeon and improve patient safety. Finally, we demonstrate the necessity of using data mining techniques to discover clinically relevant knowledge.

\section{Abbreviations}

AVF: Arteriovenous fistula

AVG: Arteriovenous graft

CDC: Centers for Disease Control and Prevention 
CKD: Chronic kidney disease

CVC: Central venous catheter

DM: Diabetes mellitus

DT: Decision tree

ESRD: End-stage renal disease

HD: Hemodialysis

HKC: Hasheminejad Kidney Center

IREP: Incremental reduced error pruning

KDD: Knowledge discovery in databases

MS: $\quad$ Master of Science

NKF: National Kidney Foundation

RIPPER: Repeated incremental pruning to produce error reduction

TDIDT: Top-down induction of decision trees

TMU: Tarbiat Modares University

TUMS: Tehran University of Medical Sciences

USRDS: United States Renal Data System

VA: Vascular access.

\section{Disclosure}

This paper is extracted from successfully defended thesis entitled "A data mining approach to fistula surgery failure analysis in hemodialysis patients" by the first author (M. Rezapour) for the degree of M.S. in IT engineering. None of the author and coauthors of this paper have any direct or indirect financial relation with the commercial identity, "Rapid Miner," mentioned in this paper.

\section{References}

[1] M. K. Zadeh, Z. Omrani, N. Najmi et al., "Prevalence and survival of hemodialysis vascular access in end-stage renal disease (ESRD) patients of Tehran, Iran," Annals of Iranian Medicine, vol. 3, no. 8, pp. 37-40, 2006.

[2] J. V. Tushar, "Dialysis vascular access selection in elderly patients," US Nephrology, vol. 6, no. 2, pp. 128-130, 2011.

[3] R. Fluck and M. Kumwenda, "UK Renal Association Clinical Practice Guidelines for Vascular Access, 2008-2011," final version, 2011, http://www.renal.org/Libraries/Guidelines/Vascular _Access_for_Haemodialysis_-_FINAL_VERSION_-_05_January 2011.sflb.ashx .

[4] B. Gerald, "Fistula first has resulted in an Increase in catheter use, 6th annual controversies in dialysis access," Journal of Vascular Access, vol. 10, pp. 284-285, 2009.

[5] G. A. Beathard, P. Arnold, J. Jackson et al., "Aggressive treatment of early fistula failure. Physician operators forum of RMS lifeline," Kidney International, vol. 64, pp. 1487-1494, 2003.

[6] U. Fayyad, G. Piatetsky-Shapiro, and P. Smyth, "From data mining to knowledge discovery in databases," AI Magazine, vol. 17, no. 3, pp. 37-53, 1996.

[7] H. C. Koh and G. Tan, "Data mining applications in healthcare," Journal of Healthcare Information Management, vol. 19, no. 2, pp. 64-72, 2005.

[8] S. Biafore, "Predictive solutions bring more power to decision makers," Health Management Technology, vol. 20, no. 10, pp. 1214, 1999.

[9] R. Bellazzi, C. Larizza, P. Magni, and R. Bellazzi, “Temporal data mining for the quality assessment of hemodialysis services," Artificial Intelligence in Medicine, vol. 34, no. 1, pp. 25-39, 2005.
[10] M. F. Bries, Modeling of hemodialysis patient hemoglobin: a data mining exploration [M.S. thesis], University of Iowa, 2007.

[11] T. Knorr, L. Schmidt Thieme, and C. Johner, "Identifying patients at risk: mining dialysis treatment data," in Cooperation in Classification and Data Analysis, pp. 131-140, Springer, Berlin, Germany, 2009.

[12] Y. Altintas, H. Gokcen, M. Ulgu et al., "Analysing interactions of risk factors according to risk levels for hemodialysis patients in Turkey: a data mining application," Gazi University Journal of Science, vol. 24, no. 4, pp. 829-839, 2011.

[13] Centers for Disease Control and Prevention (CDC), National Chronic Kidney Disease Fact Sheet: General Information and National Estimates on Chronic Kidney Disease in the United States, 2010, U.S. Department of Health and Human Services (HHS), CDC, Atlanta, Ga, USA, 2010.

[14] National Kidney Foundation (NKF), “The Facts About Chronic Kidney Disease (CKD)," National Kidney Foundation, 2012, http://www.kidney.org/kidneydisease/aboutckd.cfm .

[15] M. Rambod and F. Rafii, "Perceived social support and quality of life in Iranian hemodialysis patients," Journal of Nursing Scholarship, vol. 42, no. 3, pp. 242-249, 2010.

[16] S. C. Smeltzer, B. G. Bare, J. L. Hinkle, and K. H. Cheever, Medical Surgical Nursing, Lippincott Williams \& Wilkins, Philadelphia, Pa, USA, 11th edition, 2008.

[17] USRDS, 2010 Annual Data Report, the USRDS end-stage renal disease incident and prevalent quarterly update, 2010, http://www.usrds.org/qtr/default.aspx .

[18] M. Aghighi, A. Heidary Rouchi, M. Zamyadi et al., "Dialysis in Iran," Iranian Journal of Kidney Diseases, vol. 2, no. 1, pp. 11-15, 2008.

[19] J. Ethier, D. C. Mendelssohn, S. J. Elder et al., "Vascular access use and outcomes: an international perspective from the dialysis outcomes and practice patterns study," Nephrology Dialysis Transplantation, vol. 23, no. 10, pp. 3219-3226, 2008.

[20] M. M. Sepehri, M. Khavanin Zadeh, M. Rezapour et al., "A data mining approach to fistula surgery failure analysis in hemodialysis patients," in Proceedings of the 18th Iranian Conference of Biomedical Engineering (ICBME '11), pp. 15-20, December 2011.

[21] W. Cohen, "Fast effective rule induction," in Proceedings of the 12th International Conference on Machine Learning (ML '95), pp. 115-123, 1995.

[22] I. H. Witten, M. Frank, and M. A. Hall, Data Mining: Practical Machine Learning Tool and Technique With Java Implementation, Morgan Kaufmann, San Francisco, Calif, USA, 3rd edition, 2011.

[23] A. B. S. Serapião and A. C. Bannwart, "Knowledge discovery for classification of three-phase vertical flow patterns of heavy oil from pressure drop and flow rate data," Journal of Petroleum Engineering, vol. 2013, Article ID 746315, 8 pages, 2013.

[24] J. Quinlan, C4.5: Programs for Machine Learning, Morgan Kaufmann, San Francisco, Calif, USA, 1993.

[25] G. Ilczuk, R. Mlynarski, A. Wakulicz-Deja, A. Drzewiecka, and W. Kargul, "Rough set techniques for medical diagnosis systems," in Proceedings of the Computers in Cardiology, pp. 837840, September 2005.

[26] P. Cohen and D. Jensen, "Over fitting explained," in Proceedings of the 6th International Workshop on Artificial Intelligence and Statistics, pp. 115-122, 1997.

[27] R. R. Bouckaert, E. Frank, M. Hall et al., WEKA Manual for Version 3-6-0, University of Waikato, Hamilton, New Zealand, 2008 . 


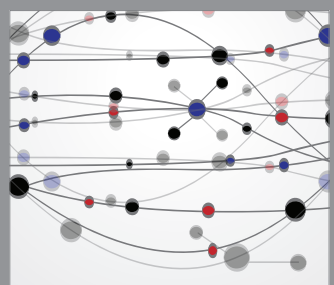

The Scientific World Journal
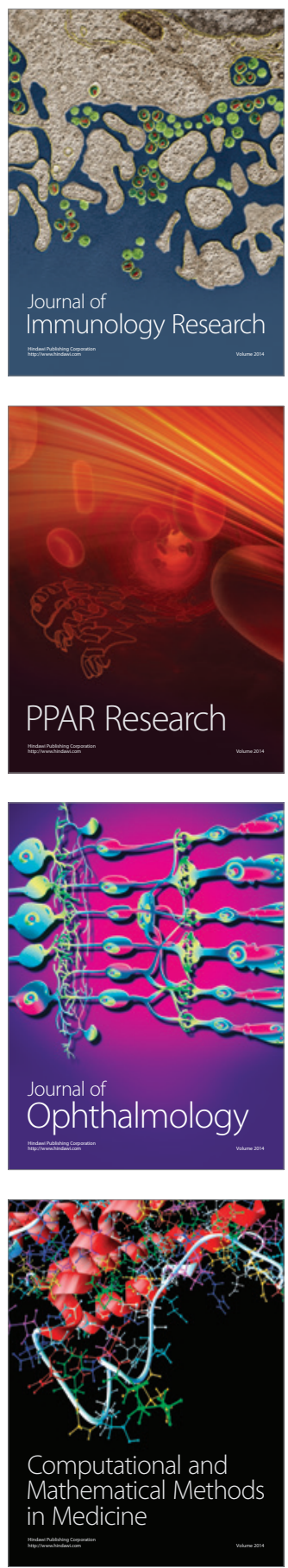

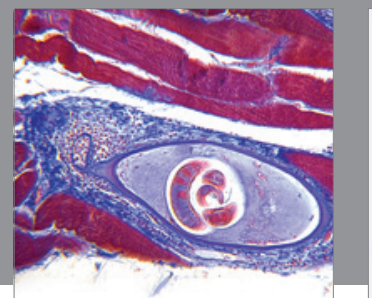

Gastroenterology

Research and Practice
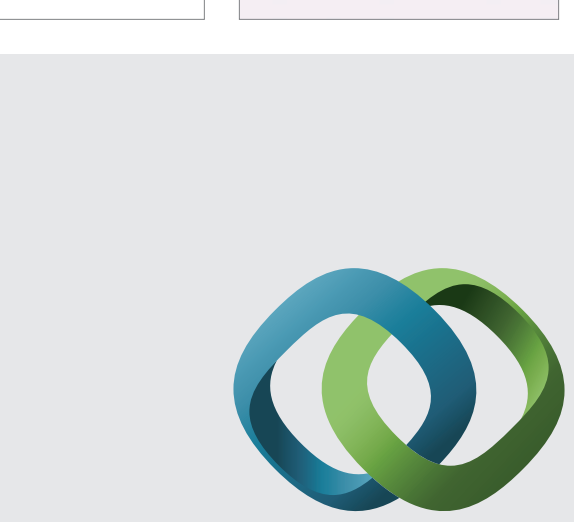

\section{Hindawi}

Submit your manuscripts at

http://www.hindawi.com
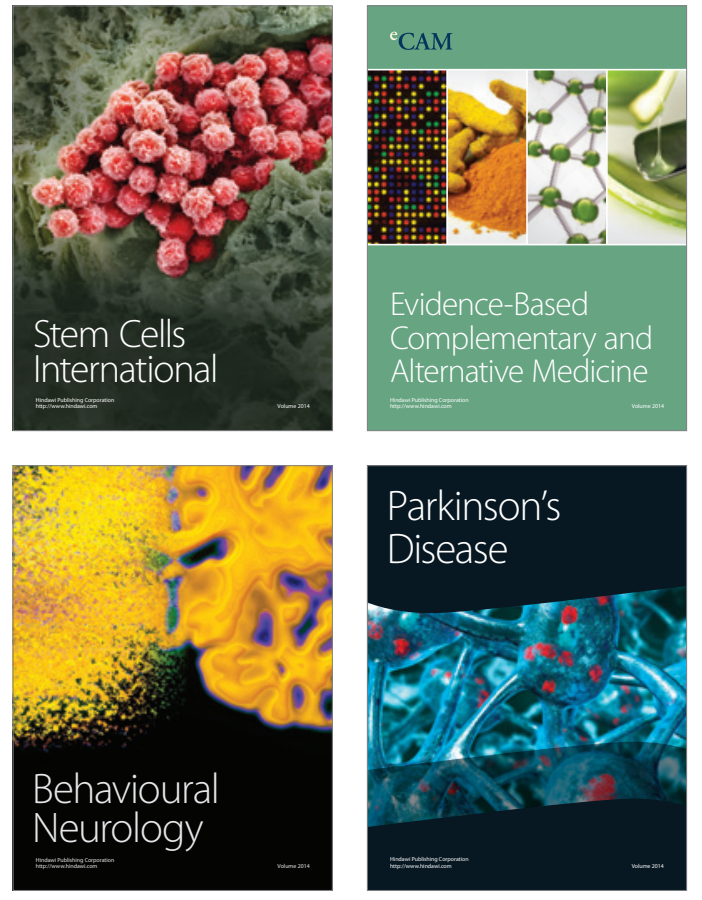
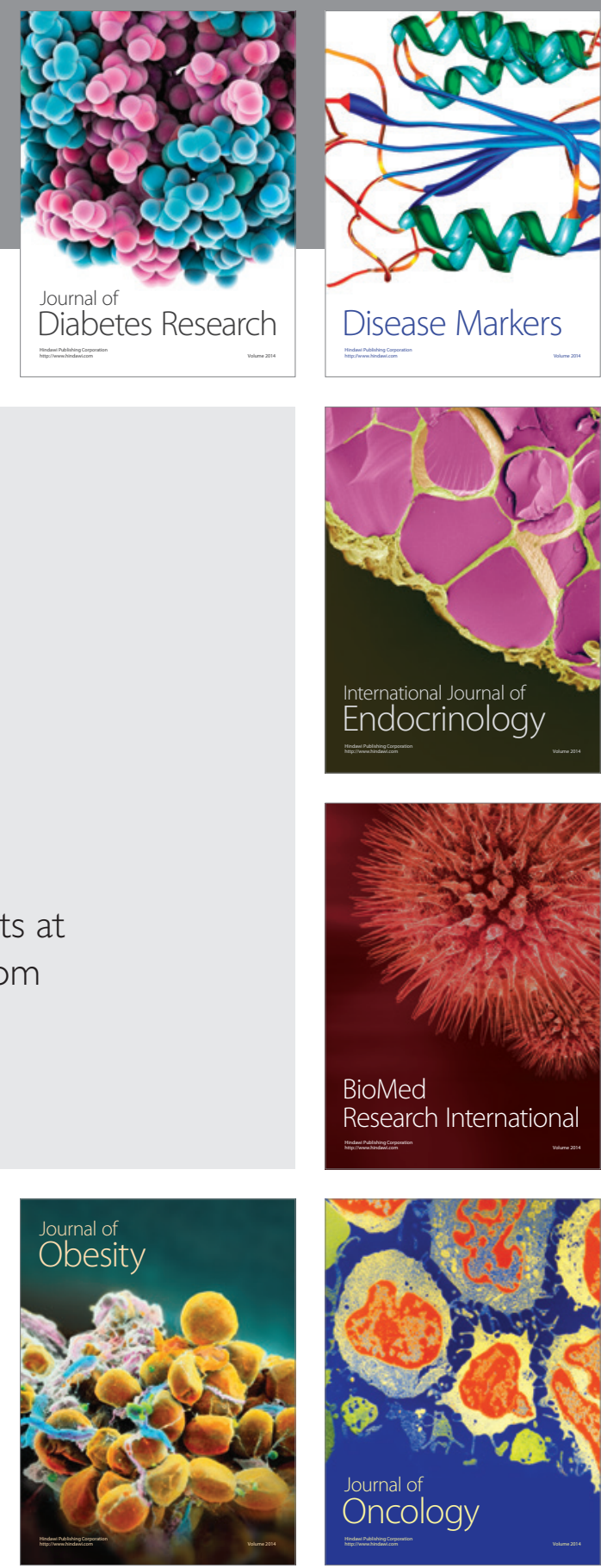

Disease Markers
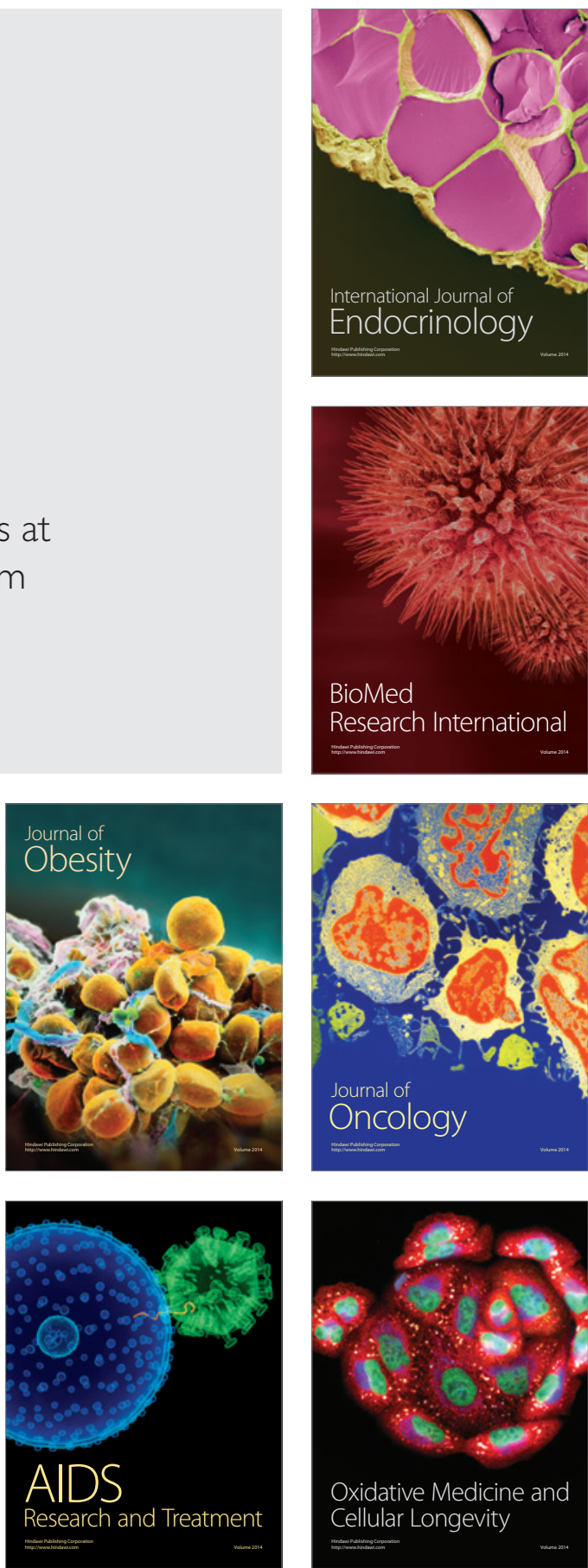